\title{
MASS CONTINUOUS COCHAINS ARE DIFFERENTIAL FORMS
}

\author{
F. J. ALMGREN, JR. ${ }^{1}$
}

Suppose $X$ is the cochain (i.e. linear function) on the linear space of 1-dimensional oriented polyhedral chains in the $(x, y)$-plane defined on 1-simplexes $\sigma$ as follows:

$$
\begin{array}{ll}
X \cdot \sigma=\int_{\sigma} d x & \text { if the slope of } \sigma \text { is rational, } \\
X \cdot \sigma=0 & \text { otherwise. }
\end{array}
$$

Unlikely as it may seem, there exists a differential 1 -form $\omega$ such that

$$
X \cdot \sigma=\int_{\sigma} \omega
$$

for each 1 -simplex $\sigma$. In particular, $\omega \cdot \vec{\sigma}$ is a bounded Hausdorff 1-dimensional measurable function along the line containing $\sigma$.

Each $k$-dimensional integrable differential form $\omega$ on $R^{n}, 0 \leqq k \leqq n$, determines a cochain $X$ on the linear space $C_{k} R^{n}$ of all $k$-dimensional oriented polyhedral chains in $\boldsymbol{R}^{n}$, namely

$$
X \cdot \sigma=\int_{\sigma} \omega
$$

for each $\sigma \in C_{k} R^{n}$. It is natural to seek conditions on an arbitrary cochain $X$ sufficient to insure the existence of such a corresponding differential form. Such conditions have usually been expressed in terms of continuity hypotheses on $X$. In [5] it was shown that to each flat cochain and to each sharp cochain there corresponds such a differential form. In [4] it was shown that to each local $L_{1} 1$-cochain in the plane there corresponds such a 1 -form. In this paper we make the observation that the existence of these differential forms as well as the form indicated in the example above follows almost immediately from the assumption of the well ordering principle and the continuum hypothesis. This observation is, in fact, an easy consequence of H. Federer's remarks on the "sigma-finite" hypothesis of the Radon-Nikodym theorem which will appear in his book on geometric measure theory. It seems useful, however, to make these

Received by the editors December 24, 1964.

1 Supported by National Science Foundation grant NSF-GP2439. 
remarks explicit in this context. Our observation was suggested by, and gives a partial answer to, a problem of $\mathrm{H}$. Whitney [5, p. 327].

Definitions. (1) For the definition of a polyhedral chain $A$ and its mass $M(A)$, see $[5$, pp. 152, 153].

(2) For the definition of a current $A$ and its mass $M(A)$, see [3, pp. 462, 463]. A $k$-dimensional current $A$ in $R^{n}$ is called a $k$ dimensional real rectifiable current (or $k$-dimensional real current) if and only if for each $\epsilon>0$ there exists a class 1 mapping $f: R^{k} \rightarrow R^{n}$ and a continuous function $g: R^{k} \rightarrow R$ such that $f \mid\{x: g(x)>0\}$ is a homeomorphism and $\boldsymbol{M}\left(A-f_{\boldsymbol{f}}\left(\boldsymbol{R}^{k} \wedge g\right)\right)<\epsilon$. Here $\boldsymbol{R}^{k} \wedge g$ defines a $k$-dimensional current in $R^{k}$ in the obvious way. Clearly each $k$-dimensional polyhedral chain in $\boldsymbol{R}^{n}$ is a $k$-dimensional real rectifiable current in a natural way. The real currents are the oriented analogues of the real varifolds $[1,5.4]$.

(3) For each $k$-dimensional real rectifiable current $A$ in $R^{n},\|A\|$ denotes the associated variation measure [3, p. 464]. Also, $A^{\rightarrow}(x)$ denotes the $k$-vector tangent to $A$ at $x$ which is defined and has norm 1 for $\|A\|$ almost all $x$ in $R^{n}\left[3\right.$, p. 495]. We define, for $x \in R^{n}$ and measure $\phi$ on $R^{n}, \odot^{k}(\phi, x)=\lim _{r \rightarrow 0}+\left(\omega_{k} r^{k}\right)^{-1} \phi\{y:|y-x|<r\}$ whenever this limit exists. Here $\omega_{k}$ is the $k$-dimensional area of the unit $k$-disk.

(4) $H_{n}^{k}$ denotes Hausdorff $k$-dimensional measure in $R^{n}$ [3, p. 493]. $H_{k}^{k}$ equals Lebesgue $k$-dimensional measure in $R^{k}$.

(5) $\mathbf{\Lambda}_{k} \boldsymbol{R}^{n}$ denotes the real vector space of $k$-vectors in $\boldsymbol{R}^{n}$, and $\mathbf{\Lambda}^{k} \boldsymbol{R}^{\boldsymbol{n}}$ denotes the real vector space of $k$-covectors in $R^{n}[3$, p. 461].

Theorem. Let $0 \leqq k \leqq n<\infty$, and suppose $C_{k} R^{n}$ denotes either (i) the real linear space of all $k$-dimensional oriented polyhedral chains in $\boldsymbol{R}^{n}$, or (ii) the real linear space of all $k$-dimensional real rectifiable currents in $\boldsymbol{R}^{n}$. Suppose $X: \boldsymbol{C}_{k} \boldsymbol{R}^{n} \rightarrow \boldsymbol{R}$ is a cochain (i.e. linear function) which is mass continuous (i.e. there exists $m<\infty$ such that $X \cdot A \leqq m M(A)$ for each $\left.A \in C_{k} R^{n}\right)$. Assume the continuous hypothesis and the well ordering principle. Then there exists a differential form $\omega: R^{n} \rightarrow \mathbf{\Lambda}^{k} R^{n}$ such that for each $A \in C_{k} R^{n}$,

$$
X \cdot A=\int_{R^{n}} \omega(x) \cdot A \rightarrow(x) d\|A\| x .
$$

ProOF.

Part 1. We will prove the theorem only in the case $C_{k} R^{n}$ is the real linear space of $k$-dimensional real rectifiable currents in $R^{n}$. The proof for polyhedral chains is similar and easier. Let then $A \subset C_{k} R^{n}$ denote the class of all $k$-dimensional connected compact oriented submani- 
folds with boundary of $\boldsymbol{R}^{n}$ of class 1 . We will construct the differential form $\omega$ so that for each $A \in A,\left(^{*}\right)$ is satisfied. It is then an easy consequence of the definition of real currents that $\left(^{*}\right)$ holds for each $A \in \boldsymbol{C}_{k} \boldsymbol{R}^{n}$. It is not difficult to verify that if $\boldsymbol{\aleph}_{0}$ denotes the cardinal number of the integers, then the cardinal number of $\boldsymbol{A}$ does not exceed $2 \aleph_{0}$. By the continuum hypothesis $2^{\aleph_{0}}=\aleph_{1}$. Using the well ordering principle, we now well order the elements $A$ of $A$ such that preceding each element of $\boldsymbol{A}$ there are at most countably many other elements of $\boldsymbol{A}$.

Part 2. Suppose $m<\infty$ and $X: C_{k} R^{n} \rightarrow R$ is linear with $X \cdot A$ $\leqq m M(A)$ for each $A \in C_{k} R^{n}$. For each $A \in A$, let $|A|$ denote all the nonboundary points of $A$. Let

$$
\begin{gathered}
f_{A}:|A| \rightarrow R, \\
f_{A}(x)=\lim _{r \rightarrow 0^{+}}\left(\omega_{k} r^{k}\right)^{-1} X \cdot(A \cap\{y:|y-x|<r\})
\end{gathered}
$$

if this limit exists

$$
f_{A}(x)=0 \text { otherwise. }
$$

One verifies that $f_{A}$ is a Baire function and

$$
X(A \wedge g)=\int_{R^{n}} g(x) f_{A}(x) d H_{n}^{k} x
$$

for each continuous $g: R^{n} \rightarrow R$.

We define

$$
\begin{aligned}
\omega: R^{n} & \rightarrow \mathbf{\Lambda}^{k} R^{n} \\
\omega(x) & =f_{A}(x) A \rightarrow(x)^{*}
\end{aligned}
$$

where $A$ is the first element of $\boldsymbol{A}$ containing $x$ and $A^{\rightarrow}(x)^{*} \in \mathbf{\Lambda}^{k} R^{n}$ is the covector dual to $A^{\rightarrow}(x)$.

Since, for each $A, B \in A$,

$$
\odot^{k}\left(H_{n}^{k} \cap(|A| \cup|B|), x\right)=1
$$

for $H_{n}^{k}$ almost all $x \in|A| \cup|B|\left[2\right.$, p. 129], we have that for $H_{n}^{k}$ almost all $x \in|A| \cap|B|$, either $f_{A}(x)=f_{B}(x)$ or $f_{A}(x)=-f_{B}(x)$ depending on orientations. Since, preceding each $A \in A$ there are at most countably many other elements of $\boldsymbol{A}$, we have that for each $A \in A$,

$$
\omega(x) \cdot A \rightarrow(x)=f_{A}(x)
$$

for $H_{n}^{k}$ almost all $x \in|A|$. The theorem follows.

REMARK. Both the integral currents $I_{k} R^{n}[3$, p. 467] and the real 
numbers $\boldsymbol{R}$ are modules over the ring of integers $\boldsymbol{Z}$. One verifies easily that each $\boldsymbol{Z}$ module homomorphism $X: I_{k} R^{n} \rightarrow R$ which is mass continuous corresponds to a differential form $\omega$ satisfying $\left(^{*}\right)$ for each $A \in I_{k} R^{n}$. There are no nontrivial mass continuous $\boldsymbol{Z}$ module homomorphisms $X: I_{k} R^{n} \rightarrow Z$.

REMARK. Our theorem implies, of course, the existence of a differential form corresponding to each flat cochain $[5$, p. 156], each sharp cochain [5, p. 160], and, by an easy extension of our argument, to each local $L^{1}$ cochain [4, p. 447]. Our result, however, says nothing about the $H_{n}^{n}$ measurability of the differential form $\omega$ (unless, of course, $k=n$ ); indeed, in our initial example, Fubini's theorem tells us that $\omega$ cannot be $H_{2}^{2}$ measurable.

\section{REFERENCES}

1. F. J. Almgren, Jr., The theory of varifolds $-A$ variational calculus in the large for the k-dimensional area integrand (to appear).

2. H. Federer, The $(\phi, k)$ rectifiable subsets of $n$ space, Trans. Amer. Math. Soc. 62 (1947), 114-192.

3. H. Federer and W. H. Fleming, Normal and integral currents, Ann. of Math. 72 (1960), 458-520.

4. V. L. Shapiro, Harmonic analysis and the theory of cochains, Bull. Amer. Math. Soc. 70 (1964), 447-467.

5. H. Whitney, Geometric integration theory, Princeton Univ. Press, Princeton, N. J., 1957.

INSTITUTE For AdVANCEd STUdY 